\title{
Immune cell transcript modules reveal leukocyte heterogeneity in synovial biopsies of seronegative spondylarthropathy patients
}

\author{
Robin M Hallett ${ }^{1}$ and Tracy Chew ${ }^{2,3^{*}}$
}

\begin{abstract}
Background: The objective of this study was to identify leukocyte cell types found within the synovia of patients with seronegative spondylarthropathies (SpA), such as ankylosing spondylitis (AS), using transcription based analyses.

Methods: Leukocyte transcriptional profiles obtained from the NCBI's gene expression omnibus and prediction analysis of microarrays (PAM) was used to identify 25-gene leukocyte metagenes. Subsequently, transcriptional profiles from murine and clinical models of AS and SpA were interrogated to characterize the local infiltration of leukocytes in SpA synovia.

Results: Analysis of a proteoglycan-induced murine model of AS reveals infiltration of dendritic cells, CD4+ T cells, monocytes, and natural killer cells to the spine. In human SpA and AS patients, transcriptional analysis of synovial biopsies revealed local infiltration of dendritic cells and CD4+ T cells.

Conclusions: We identified leukocyte cell types that infiltrated the synovial of SpA patients. Our results imply a role for dendritic cells and CD4+ T cells in the local inflammation that underlies pathogenesis in patients with SpA.

Keywords: Spondylarthropathies, Ankylosing spondylitis, Immune infiltration, Inflammation, Rheumatology, Leukocyte, Transcriptional analysis, Gene signature, Metagene, Computational biology
\end{abstract}

\section{Background}

Spondyloathropathies $(\mathrm{SpA})$ represent a spectrum of inflammatory and degenerative joint diseases affecting the spine, with ankylosing spondylitis (AS) being among the most prevalent and debilitating worldwide. Primary manifestations of SpA involve axial manifestations such as sacroiliitis, and include both musculoskeletal and extraarticular symptoms. AS in particular is characterized by a variety of inflammatory conditions including joint and back pain, with severe cases progressing to spinal fusion and kyphosis leading to restricted mobility. While the disease is known to be inflammatory in nature, the specific mechanisms and cell types triggering inflammation within the joint are less well characterized.

\footnotetext{
* Correspondence: tchew@cmcc.ca

${ }^{2}$ Department of Graduate Education and Research, Canadian Memorial Chiropractic College, 6100 Leslie Street, Toronto, Ontario M2H 3J1, Canada ${ }^{3}$ Department of Pathology and Microbiology, Canadian Memorial Chiropractic College, 6100 Leslie Street, Toronto, Ontario M2H 3J1, Canada Full list of author information is available at the end of the article
}

Several genome-wide association studies and gene expression studies have been performed in AS and SpA patients, revealing several lines of evidence regarding the mechanisms of inflammation underlying disease [1-3]. Importantly, several important cellular processes have been implicated in the immune-mediated pathogenesis of AS, including antigen processing, the tumor necrosis factor alpha $(\mathrm{TNF} \alpha) /$ nuclear factor kappa beta $(\mathrm{NF} \kappa \mathrm{B}) /$ interleukin 1 beta (IL-1 $\beta$ ) axis, and the IL-23/IL-17/T helper 17 (Th17) axis. Serum TNF $\alpha$ and IL-6 levels correlate positively with disease [4], and TNF $\alpha$ inhibitors are the primary course of treatment for AS patients. In addition, the association of $I L 12 B$ and $I L 23 R$ genes in AS patients, as well as elevated serum and synovial levels of IL-17related gene products suggests a role for the Th17/IL-23/ IL-17 axis in pathology [5]. Importantly, these observations were derived from serological studies, and do not delineate local changes in a joint that may directly reflect important inflammatory processes underlying disease. 
More importantly, it is unclear what inflammatory processes specifically occur within the synovia that are associated with joint/spinal inflammation, and which cell types are responsible for inflammatory cytokine production. To date, only a handful of studies have implicated a role for specific immune cell types involved in the inflammatory response in the joints of AS patients. The detection of FoxP3+ CD4+ T cells, IL-17-producting $\mathrm{T}$ cells, and KIR3DL2+ Th17 cells in the synovial fluid of patients with AS and with SpA implies a role for regulatory T cells in the local inflammatory processes in the joint [6-8]. Additionally, some early evidence of local infiltration of mast cells and phagocytic monocytes in the knee of AS patients by microscopy implies a role for these immune cell types in the local tissue [9]. However, this observation has not been validated by more comtemporary quanititative measures such as flow cytometry. Additional cells types associated with inflammation have been identified in the peripheral blood, but fail to delineate a relationship to the local inflammatory milieu [5,10-13].

Importantly, gene expression profiles have been conducted from the synovial fluid of AS patients and from mouse models of AS. Given the availability of gene expression profiles from purified leukocyte populations as well as from the synovial biopsies of AS and SpA patients, we sought to identify the immune cell subtypes infiltrating the local inflammatory milieu of these patients.

\section{Methods}

\section{Patients and samples}

All data were publicly available and downloaded from the gene expression omnibus (GEO, http://www.ncbi.nlm.nih. gov/geo/). The leukocyte (GSE1133 \& GSE22886), mouse AS (GSE41039) and patient biopsy (GSE41038) datasets were downloaded and processed independently [14-17]. For Affymetrix data, the raw .CEL files comprising each dataset were download and normalized using the Robust Multichip Algorithm (RMA) to generate probe set intensities [18]. For Illumina data, normalized data was downloaded directly from GEO. All human data were downloaded from a publicly available database, and were previously published. No new patients or samples were collected for this study.

\section{Prediction analysis of microarrays (PAM)}

PAM was downloaded as an excel add-in from http:// www-stat.stanford.edu/ tibs/PAM/, and was used according to the available manual. Briefly, we used this algorithm to interrogate the human leukocyte series for the top 25 class genes where each different population of purified leukocytes was an independent class. Leukocyte class assignment was made as follows: Monocytes (GSM18871 \& 18872), DCs (GSM18873 \& 18874), NK cells (GSM18875 \& 18876), CD4+ $\mathrm{T}$ cells (GSM18877 \& 18878), CD8+
T cells (GSM18879 \& 18880), and B cells (GSE18881 \& 18882).

\section{Evaluation of leukocyte metagenes}

For cross-platform comparisons, datasets were collapsed to single genes based on highest mean average and mapped based on Unigene ID. To evaluate leukocyte metagenes, the expression values for each gene was transformed such that the mean and standard deviation were set to 0 and 1 in each dataset, respectively. A metagene score was calculated for each sample as follows:

$$
\frac{\sum_{i \in p} x_{i}}{n_{p}}
$$

where $x$ is the transformed expression, $n$ is the number of genes in class set $\mathrm{P}$ and $\mathrm{P}$ is the set of class marker genes for a particular metagene [19-21].

\section{Statistical analysis}

T-tests were used to compare indices between control and spondylarthropathy samples in both murine and human datasets. All tests were two-sided and a p-value of 0.05 or less was considered statistically significant.

\section{Results}

\section{Identification of leukocyte expression metagenes}

Whereas the underlying mechanisms behind AS and other $\mathrm{SpA}$ are known to be inflammatory in nature, the specific leukocyte cell populations that drive inflammation are unknown. Hence, we sought to measure infiltration of specific leukocyte cell populations within synovial biopsies of experimental and clinical models of AS and SpA. To this end, we identified leukocyte transcript modules, which could be implemented to measure leukocyte infiltration in patient biopsies for which the global gene expression profile is known. Based on publicly available global gene expression profiling data of the human transcriptome, we identified samples representing multiple purified leukocyte cell populations including monocytes, dendritic cells (DC), B cells, natural killer (NK) cells, CD4+ T cells and CD8+ T cells. We used PAM [22] to identify a gene expression based classifier that could discriminate between leukocyte cell type according to patterns in gene expression. Briefly, PAM is an efficient tool to identify molecular patterns that is applicable to gene expression data, and uses nearest shrunken centroids to identify subsets of genes that best characterize sample classes. We used PAM to identify a 150 gene classifier that comprised the top 25 marker genes associated with each leukocyte cell type (see Additional file 1: Table S1). This composite gene signature is known as a metagene, and gene clusters representing each leukocyte metagene is shown in Figure 1A. To test whether the expression of each leukocyte metagene was highly 


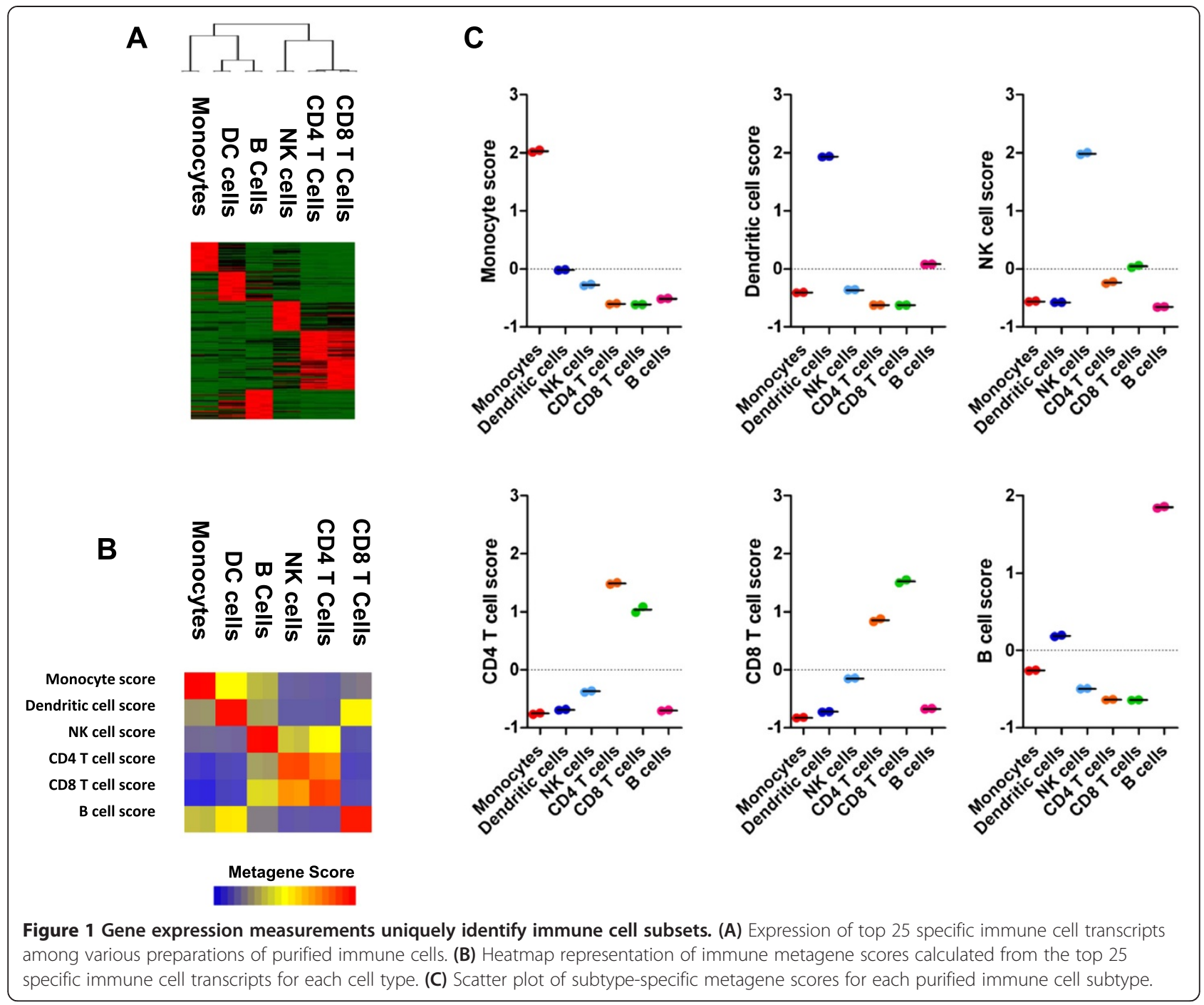

discriminatory between specific populations of leukocytes, we calculated immune cell metagene scores for each cell type across all cell types (Figures $1 \mathrm{~B}$ and $\mathrm{C}$ ). For example, the average monocyte metagene score was highest in the purified monocyte cell population, and negative in each other leukocyte population. Although we observed some cross-specificity between the CD4 and CD8 T cell signatures, the leukocyte metagene score was highest in the target cell population for each leukocyte cell type, supporting the overall validity of the approach. To further validate this approach we downloaded additional independent gene expression profiles from purified leukocyte populations and tested the capacity of the various metagenes to accurately discriminate between the cell types [17]. Importantly, the metagene signatures were sufficient to stratify these various leukocyte cell types (see Additional file 2: Figure S1). Based on these data, we concluded that leukocyte metagene signatures could be implemented to measure infiltration of specific leukocyte cell populations in patient biopsies.

\section{Leukocyte metagenes identify local infiltration of multiple} leukocyte subsets in a murine model of AS

Although the overarching objective of this work was to was identify populations of leukocytes that might mediate inflammation in human patients with either AS or SpA, we first sought to examine the expression of leukocyte metagenes in a mouse model of AS. In this case, the mouse model offers several advantages over the human samples, including samples being harvested from mice with identical genetic backgrounds, as well as whole spine samples being obtained for global gene expression profiles. Briefly, we downloaded publicly available gene expression profiles of control $(n=4)$ and experimental $(n=4)$ mouse spines based on the proteoglycan-induced AS model in BALB/c mice [23]. We observed expression differences between 
control and experimental mice for several of the leukocyte metagenes (Figure 2A). Specifically, we observed increased monocyte, DC, NK, and CD4+ T cell metagene expression within the spines of mice induced with experimental AS (Figure 2B). Hence, we conclude that in an experimental mouse model of AS, the disease is associated with increased monocyte, DC, NK cells, and CD4+ T cell infiltration relative to control animals.

\section{Leukocyte metagenes identify differential leukocyte infiltration in synovial biopsies of patients with seronegative SpA}

To extend these findings in human patients, we examined the expression of leukocyte metagenes in synovial biopsies extracted from patients with seronegative SpA or in control biopsies. Briefly, we downloaded publicly available gene expression profiling data for the samples described above, and compared leukocyte metagene expression in biopsies derived from the $8 \mathrm{SpA}$ patients (2 AS, $6 \mathrm{SpA}$ ) with 7 control biopsies ( 4 normal, 3 OA). Similar to the mouse model data, we observed differences in leukocyte metagene expression among the control and SpA patient biopsies (Figure 3A). Specifically, we observed increased
DC and CD4+ T cell metagene expression in the SpA biopsies relative to control biopsies (Figure $3 \mathrm{~B}$ ). Interestingly, we did not observe increased expression of monocyte and NK cell metagene expression in the human SpA biopsies relative to control, whereas these metagenes were significantly more highly expressed in experimental mouse biopsies. A post-hoc analysis of individual genes comprising the differentially expressed leukocyte metagenes, revealed that metagenes were upregulated as a whole, and the observed increases were not driven by dramatic up-regulation relatively few genes (see Additional file 3: Figure S2). Importantly, this suggests that our observations are not confounded by dramatic changes in single genes, and likely represent true measurements of infiltration.

Taken together with our previous data, these findings strongly suggest that the joints of patients with AS/SpA contain increased leukocyte infiltrate that primarily comprises $\mathrm{DC}$ and CD4+ $\mathrm{T}$ cells relative to control patient joints.

\section{Discussion}

This study identifies infiltrating leukocyte subtypes within the local tissue of patients suffering from seronegative

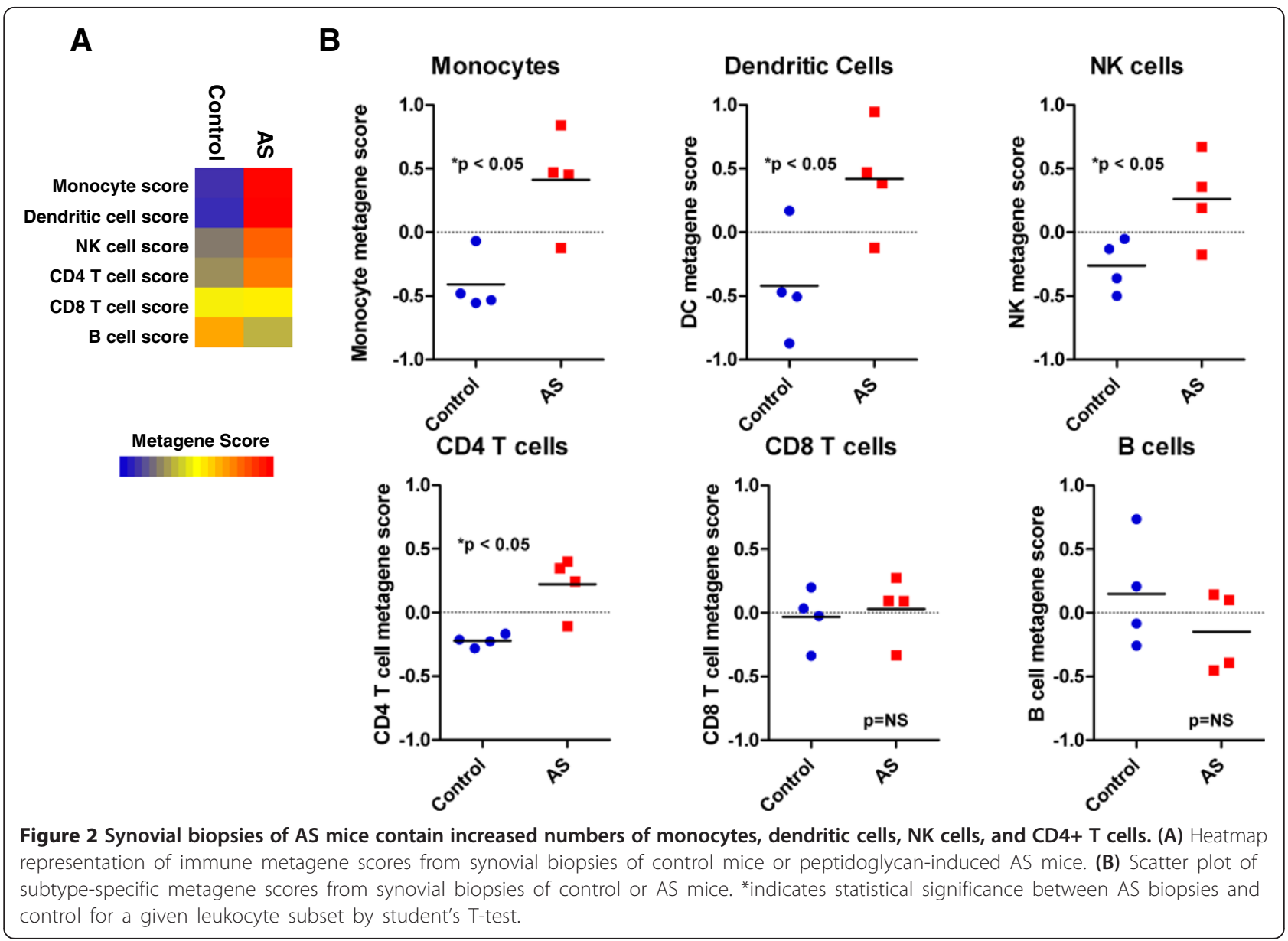




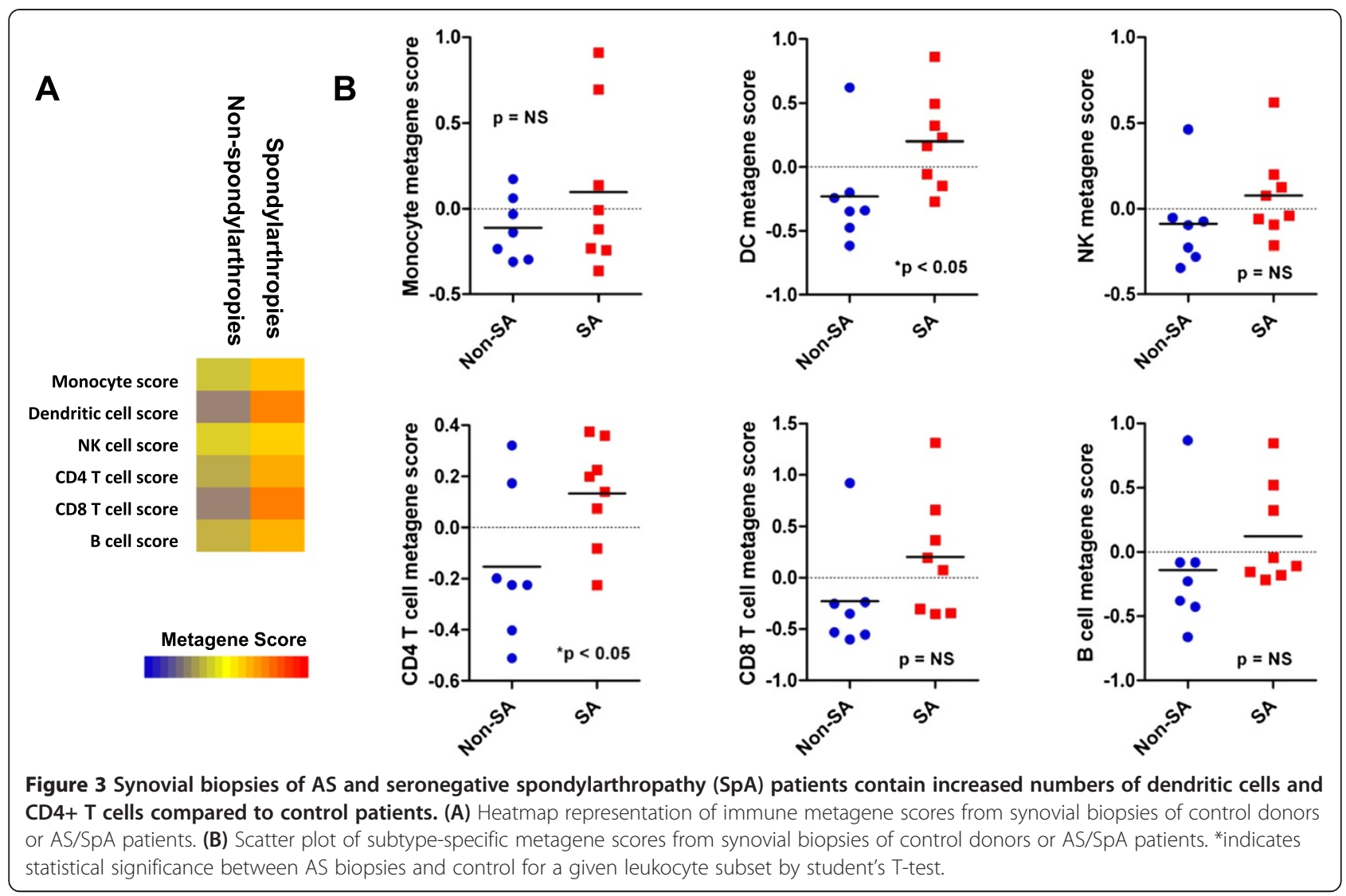

SpA. To our knowledge, this is the first study to relate leukocyte cell types found in peripheral blood to their infiltration within the local inflammatory milieu of the joints in these patients. Previous studies identified increased numbers of IL-17- and IL-22-producing CD4+ T cells [5,10], but their specific role in the pathogenesis of disease remains unclear. Furthermore, their presence within the synovial of inflamed joints in the spine has not been elucidated. As gene expression profiles of specific $\mathrm{T}$ cell subsets, including Th17, $\gamma / \delta$, and regulatory $\mathrm{T}$ cells, is not available, this study is not able to distinguish these specific T cell subsets. An interrogation of these cell types in the local tissue would be an important follow-up study, as a number of $\mathrm{T}$ cell subsets have been implicated in disease from peripheral blood analyses.

Previous studies also identified various biomarkers in the peripheral blood of AS patients that correlate with disease. For example, Bal et al. [4] demonstrate that AS patients have increased circulating levels of TNF $\alpha$; interestingly, monocyte-derived DCs and macrophages are an important source of TNF $\alpha$ and play a crucial role in the initiation of a local inflammatory response. Additionally, several TNF $\alpha$-related genes have been identified in the positively correlated immune cell subsets, implying an important role for this pathway in immune pathogenesis of disease. Although we did not observe significantly increased expression of monocyte markers in SpA patients, a proportion of the patients in this sample set did exhibit increased monocyte metagene expression. Whether this trend would be statistically significant in a larger sample size is unclear. Together with the observation that monocytes were found at increased levels in a mouse model of AS, it is clear that infiltrating monocytic cells including monocyte-derived DCs are associated with the disease state. Similarly, we have shown here that professional antigen presenting cells including monocytederived cells and DCs are upregulated in AS synovial, as previously observed in peripheral blood. Interestingly, DCs have been shown to be a source of elevated serum ERAP1 expression in AS patients, implying a dysfunctional role of DCs in antigen presentation leading to inflammation [24]. Finally, the IL-23/Th17/IL-17 axis has been implicatied in AS pathogenesis [1-3]. Here, we report increased levels of $\mathrm{CD} 4+\mathrm{T}$ cells in within the synovial fluid of AS/SpA patients. Whether these cells also express IL-17, or include Th17 cells, is not clear, as transcriptional profiles of purified Th17 cells are not available for metaanalysis of this type.

Importantly, immune pathways previously identified to underlie AS pathogenesis have not been identified in the local environment in our analysis. For example, Baek et al. observed increased levels of IL-4+ CD8+ T cells in 
the peripheral blood of AS patients [10]. In our study, we do not observe CD8+ T cells levels within the synovia, implying that this cell type may not play as important a role in the local tissue as it does in the periphery. Additionally, a recent identification of abzymes (antibodies with catalytic activity) has been correlated to SpA [25], despite the absence of B cells within the local tissue. Indeed, extra-articular symptoms of AS and other SpA may involve such cell types; whether these cell types infiltrate the local environment of an extra-articular site of inflammation is an important question and remains unclear.

\section{Conclusions}

Our data suggest that local inflammatory processes underlying SpA cannot be deduced based on biomarkers identified in peripheral blood. While peripheral blood markers serve as important diagnostic tools, the local environment is responsible to eliciting inflammation within the joint and should be the basis for pharmacological interventions. Therefore, a closer investigation of the local inflammatory responses underlying a variety of inflammatory arthritic diseases, including $\mathrm{AS} / \mathrm{SpA}$, is an important first step in understanding and targeting pathways involved in pathogenesis of disease.

\section{Additional files}

Additional file 1: Table S1. Leukocyte metagene composition. Top 25 genes comprising the leukocyte gene signature for monocytes, dendritic cells, natural killer cells, CD4+ T cells, CD8+ T cells, and B cells,

Additional file 2: Figure S1. Validation of immune cell metagenes using independent data. Metagene scores for immune cell subtypes using an independent gene expression dataset.

Additional file 3: Figure S2. Gene-centric evaluation of differentially expressed leukocyte metagenes in the mouse (A-D) and human (E \& F) datasets. Description of data: Heatmap representation of gene expression in cell types identified in this study. Genes comprising the leukocyte metagene were analyzed for differential expression between healthy and diseases, and indicate no clear pattern of single gene-driven differences in metagene scores.

\section{Competing interests}

The authors declare that they have no competing interests.

\section{Authors' contributions}

$\mathrm{RMH}$ was responsible for study conception and design, data analysis, and statistical analysis. TC was responsible for study conception and design, and preparation of the manuscript. All authors read and approved the final manuscript.

\section{Acknowledgements}

The authors would like to thank Dr. Carlo Ammendolia and Dr. Brian Budgell for helpful discussions. TC is funded by the Department of Graduate Education and Research at CMCC.

\section{Author details}

${ }^{1}$ Path Genomics, Hamilton, Ontario, Canada. ${ }^{2}$ Department of Graduate Education and Research, Canadian Memorial Chiropractic College, 6100 Leslie Street, Toronto, Ontario M2H 3J1, Canada. ${ }^{3}$ Department of Pathology and
Microbiology, Canadian Memorial Chiropractic College, 6100 Leslie Street, Toronto, Ontario M2H 3J1, Canada.

Received: 26 June 2014 Accepted: 16 December 2014 Published: 19 December 2014

\section{References}

1. Reveille JD, Sims AM, Danoy P, Evans DM, Leo P, Pointon JJ, Jin R, Zhou X, Bradbury LA, Appleton LH, Davis JC, Diekman L, Doan T, Dowling A, Duan R, Duncan EL, Farrar C, Hadler J, Harvey D, Karaderi T, Mogg R, Pomeroy E, Pryce K, Taylor J, Savage L, Deloukas P, Kumanduri V, Peltonen L, Ring SM, Australo-Anglo-American Spondyloarthritis Consortium (TASC), et al: Genome-wide association study of ankylosing spondylitis identifies non-MHC susceptibility loci. Nat Genet 2010, 42(2):123-127.

2. Burton PR, Clayton DG, Cardon LR, Craddock N, Deloukas P, Duncanson A, Kwiatkowski DP, McCarthy MI, Ouwehand WH, Samani NJ, Todd JA, Donnelly P, Barrett JC, Davison D, Easton D, Evans DM, Leung HT, Marchini $J$, Morris AP, Spencer CC, Tobin MD, Attwood AP, Boorman JP, Cant B, Everson U, Hussey JM, Jolley JD, Knight AS, Wellcome Trust Case Control Consortium, Australo-Anglo-American Spondylitis Consortium (TASC), et al: Association scan of 14,500 nonsynonymous SNPs in four diseases identifies autoimmunity variants. Nat Genet 2007, 39(11):1329-1337.

3. Evans DM, Spencer CC, Pointon JJ, Su Z, Harvey D, Kochan G, Oppermann U, Dilthey A, Pirinen M, Stone MA, Appleton L, Moutsianas L, Leslie S, Wordsworth T, Kenna TJ, Karaderi T, Thomas GP, Ward MM, Weisman MH, Farrar C, Bradbury LA, Danoy P, Inman RD, Maksymowych W, Gladman D, Rahman P, Morgan A, Marzo-Ortega H, Bowness P, Spondyloarthritis Research Consortium of Canada (SPARCC), et al: Interaction between ERAP1 and HLA-B27 in ankylosing spondylitis implicates peptide handling in the mechanism for HLA-B27 in disease susceptibility. Nat Genet 2011, 43(8):761-767.

4. Bal A, Unlu E, Bahar G, Aydog E, Eksioglu E, Yorgancioglu R: Comparison of serum IL-1 beta, sIL-2R, IL-6, and TNF-alpha levels with disease activity parameters in ankylosing spondylitis. Clin Rheumatol 2007, 26(2):211-215.

5. Shen H, Goodall JC, Hill Gaston JS: Frequency and phenotype of peripheral blood Th17 cells in ankylosing spondylitis and rheumatoid arthritis. Arthritis Rheum 2009, 60(6):1647-1656

6. Appel H, Wu P, Scheer R, Kedor C, Sawitzki B, Thiel A, Radbruch A, Sieper J, Syrbe U: Synovial and peripheral blood CD4 + FoxP3+ T cells in spondyloarthritis. J Rheumatol 2011, 38(11):2445-2451.

7. Appel H, Maier R, Wu P, Scheer R, Hempfing A, Kayser R, Thiel A, Radbruch A, Loddenkemper C, Sieper J: Analysis of IL-17+ cells in facet joints of patients with spondyloarthritis suggests that the innate immune pathway might be of greater relevance than the Th17-mediated adaptive immune response. Arthritis Res Ther 2011, 13(3):R95.

8. Bowness P, Ridley A, Shaw J, Chan AT, Wong-Baeza I, Fleming M, Cummings F, McMichael A, Kollnberger S: Th17 Cells Expressing KIR3DL2+ and Responsive to HLA-B27 Homodimers Are Increased in Ankylosing Spondylitis. J Immunol 2011, 186(4):2672-2680.

9. Freemont AJ, Denton J: Disease distribution of synovial fluid mast cells and cytophagocytic mononuclear cells in inflammatory arthritis. Ann Rheum Dis 1985, 44(5):312-315.

10. Baek HJ, Zhang L, Jarvis LB, Gaston JSH: Increased IL-4+ CD8+ T cells in peripheral blood and autoreactive CD8+ T cell lines of patients with inflammatory arthritis. Rheumatology (Oxford) 2008, 47(6):795-803.

11. Jandus C, Bioley G, Rivals J-P, Dudler J, Speiser D, Romero P: Increased numbers of circulating polyfunctional Th17 memory cells in patients with seronegative spondylarthritides. Arthritis Rheum 2008, 58(8):2307-2317.

12. Jansen DTSL, Hameetman M, van Bergen J, Huizinga TWJ, van der Heijde D, Toes REM, van Gaalen FA: IL-17-producing CD4+ T cells are increased in early, active axial spondyloarthritis including patients without imaging abnormalities. Rheumatology 2014. 263-272. doi:10.1093/rheumatology/ keu382.

13. Kenna TJ, Davidson SI, Duan R, Bradbury LA, McFarlane J, Smith M, Weedon H, Street S, Thomas R, Thomas GP, Brown MA: Enrichment of circulating interleukin-17-secreting interleukin-23 receptor-positive $\gamma / \delta$ T cells in patients with active ankylosing spondylitis. Arthritis Rheum 2012, 64(5):1420-1429.

14. Haynes KR, Pettit AR, Duan R, Tseng H-W, Glant TT, Brown MA, Thomas GP: Excessive bone formation in a mouse model of ankylosing spondylitis is associated with decreases in Wnt pathway inhibitors. Arthritis Res Ther 2012, 14(6):R253. 
15. Su Al, Wiltshire T, Batalov S, Lapp H, Ching KA, Block D, Zhang J, Soden R, Hayakawa M, Kreiman G, Cooke MP, Walker JR, Hogenesch JB: A gene atlas of the mouse and human protein-encoding transcriptomes. Proc Natl Acad Sci 2004, 101(16):1-6.

16. Thomas GP, Duan R, Pettit AR, Weedon H, Kaur S, Smith M, Brown MA: Expression profiling in spondyloarthropathy synovial biopsies highlights changes in expression of inflammatory genes in conjunction with tissue remodelling genes. BMC Musculoskelet Disord 2013, 14(1):354

17. Abbas AR, Baldwin D, Ma Y, Ouyang W, Gurney A, Martin F, Fong S, van Lookeren Campagne M, Godowski P, Williams PM, Chan AC, Clark HF: Immune response in silico (IRIS): immune-specific genes identified from a compendium of microarray expression data. Genes Immun 2005, 6(4):319-331.

18. Irizarry RA, Hobbs B, Collin F, Beazer-Barclay YD, Antonellis KJ, Scherf U, Speed TP: Exploration, normalization, and summaries of high density oligonucleotide array probe level data. Biostatistics 2003, 4(2):249-264.

19. Hallett RM, Dvorkin-Gheva A, Bane A, Hassell JA: A gene signature for predicting outcome in patients with basal-like breast cancer. Sci Rep 2012, 2:227.

20. Hallett RM, Dvorkin A, Gabardo CM, Hassell JA: An algorithm to discover gene signatures with predictive potential. J Exp Clin Cancer Res 2010, 29(1):120.

21. Hallett RM, Pond G, Hassell JA: A target based approach identifies genomic predictors of breast cancer patient response to chemotherapy. BMC Med Genomics 2012, 5(1):16.

22. Tibshirani R, Hastie T, Narasimhan B, Chu G: Diagnosis of multiple cancer types by shrunken centroids of gene expression. Proc Natl Acad Sci U S A 2002, 99(10):6567-6572.

23. Glant TT, Mikecz K, Arzoumanian A, Poole AR: Proteoglycan-induced arthritis in BALB/c mice. Clinical features and histopathology. Arthritis Rheum 1987, 30(2):201-212.

24. Campbell EC, Fettke F, Bhat S, Morley KD, Powis SJ: Expression of MHC class I dimers and ERAP1 in an ankylosing spondylitis patient cohort. Immunology 2011, 133(3):379-385.

25. Kundzer AV, Volkova MV, Bogdanos DP, Rödiger S, Schierack P, Generalov I, Nevinsky GA, Roggenbuck D: Deoxyribonuclease activity of polyclonal IgGs: a putative serological marker in patients with spondyloarthritides. Immunol Res 2013, 56(2):457-464.

doi:10.1186/1471-2474-15-446

Cite this article as: Hallett and Chew: Immune cell transcript modules reveal leukocyte heterogeneity in synovial biopsies of seronegative spondylarthropathy patients. BMC Musculoskeletal Disorders 2014 15:446.

\section{Submit your next manuscript to BioMed Central and take full advantage of:}

- Convenient online submission

- Thorough peer review

- No space constraints or color figure charges

- Immediate publication on acceptance

- Inclusion in PubMed, CAS, Scopus and Google Scholar

- Research which is freely available for redistribution 